\title{
Modeling Analysis for the effect of night environment on athlete's psychological intervention
}

\author{
Liu ChunSheng \\ (Linyi University, Linyi Shandong 276400,China)
}

Keywords: psychological intervention; night environment; athletes;

\begin{abstract}
: the analysis of the effects of night environment on athlete's psychological intervention has a momentous significance for promoting the performance of athletes. The traditional algorithm not fully considers the instantaneous and disorder of night environmental psychological change, resulting in the reduced accuracy of analyzing the effect of psychological intervention. Therefore, a modeling method for the influence of night environment on athlete's psychological intervention based on the constrained fuzzy clustering algorithm is proposed. Firstly, the objective function of night environmental factors fuzzy clustering in the course of athletes in the sports is obtained to minimized transform. The weight and corresponding confidence coefficient of different nighttime environment factors are calculated, to establish the relationship model between night environmental factors and psychological intervention effect. The experimental results show that using the improved algorithm analyze the influence of night environment on athlete's psychological intervention, can improve the achievements of athletes at night.
\end{abstract}

\section{Introduction}

With the continued improvement of sports training skills, athlete's achievement enhancement becomes an indispensable part of the field of sports [1], but the resulting problems are increasingly serious [2]. In our country, athlete's achievement in the night is not ideal [3], it has an inevitable connection with the influence of nighttime environment on athletes [4]. Therefore, it is extremely necessary to analyze the effects of night environment for athlete's psychological intervention, to provide an accurate basis for the necessary psychological intervention for athletes [5], improving the sports performance. Therefore, the modeling analysis method for the effect of night environment on athlete's psychological intervention, has become the key subject to study in sports field [6]. At present, the main analytical methods are based on the neural network algorithm, the decision tree algorithm [7] and association rules algorithm [8]. Among them, the most commonly used is based on association rules algorithm. As the modeling analysis method for the effect of night environment on athlete's psychological intervention has an irreplaceable role in the field of sports, it has very broad prospects for development, and become the focus study of many experts.

\section{2 the theory of modeling analysis method for the effect of night environment on athlete's psychological intervention}

\subsection{Constraint fuzzy clustering processing of night environment factor}

Setting the number of night environmental factors can be described by $q$, one of the night environment factors is randomly selected, its included amount of information can be described by $u$, and to meet the constraint condition $\sum_{l=1}^{u} y_{l}=1$. Using the following formula, objective function of constrained fuzzy clustering of the night environment factors can be calculated:

$$
L(w, x, y)=\sum_{k=1}^{e} \sum_{m=1}^{q} \sum_{l=1}^{u} w_{k m}^{p} y_{l}^{\delta}\left(z_{m l}-x_{k l}\right)^{2}
$$

In the above formula, $\sum_{v=1}^{e} w_{k v}=1,1 \leq k \leq e, 1 \leq l \leq p$.

The above clustering objective function of night environment factors is made minimization 
processing, to obtain the following results:

$$
\begin{aligned}
& y_{k l}=\sum_{v=1}^{q} w_{k m}^{p} z_{m l} / \sum_{v=1}^{q} w_{k m}^{p} \\
& w_{k m}=\left(\sum_{l=1}^{u} y_{l}^{\delta} \| z_{m l}-\left.x_{k l}\right|^{1 /(1-p)} \times\left(\sum_{v=1}^{e}\left(\sum_{l=1}^{u} y_{l}^{\delta}\left\|z_{m l}-x_{k l}\right\|\right)^{1 /(1-p)}\right)^{-1}\right. \\
& y_{l}=F_{l}^{1 /(1-\delta)}\left(\sum_{v=1}^{p} F_{v}^{1 /(1-\delta)}\right)^{-1} F_{l}=\sum_{k=1}^{e} \sum_{m=1}^{q} w_{k m}^{p}\left(z_{m l}-x_{k l}\right)^{2}
\end{aligned}
$$

In the clustering processing of the night of environmental factors, it needs to obtain the ratio and fuzzy index of each night environment factors, using this method can make the acquisition results to meet the requirements of the objective function. According to the following steps, it can make detailed description of the realization process of constrained fuzzy clustering:

(1) According to the number of night environmental factors clustering transform, ratio of fuzzy index and night environment factors, the matrix of night environment factors and the corresponding ratio can be obtained.

(2) Using the ratio of night environmental factors, can obtain the objective function values. In the process of clustering processing, assuming the iteration number is more than the set threshold value, then the operation is ended; otherwise, operation is continued.

(3) In the clustering process of the night environmental factors, the deviation will be occurred, it needs to compensate for the deviation.

(4) According to the correction cluster center of night environmental factors, the membership function can be made update processing.

(5) The matrix of the ratio of night environmental factors is made update processing.

According to the above method, it can make constrained fuzzy clustering process of night environment factors, so as to provide accurate data base for the modeling of night environment factors and athlete's psychological intervention effect.

2.2 establishment of relationship model of night environment factors and psychological intervention effect

According to night environment factors to constrain fuzzy clustering results, the relationship model of night environment factors and psychological intervention effect can be established, so as to realize analysis for relation. The following steps can be described in detail:

Assuming the collection constituted by all night environment factors can be described by $Z$, the quantity of all elements in the collection can be depicted by $q$, the number of corresponding attributes can be described by $p$. The following formula is used to obtain the corresponding support ratio:

$$
H(D)=\sum_{l=1}^{q} \prod \omega\left(d_{k}, z_{l}\right)
$$

The above formula, $q$ is used to describe the number of all-night environmental factors, $\omega$ is used to describe the corresponding membership of night environmental factors, $\omega\left(d_{k}, z_{l}\right)$ is used to describe the membership of $l$ - th elements in the $d_{k}$.

The following formula is utilized to get confidence of any one night environment factors:

$$
H^{\prime}=\frac{\sum_{k=1}^{r}\left[\Pi \omega\left(f_{k}, z_{l}\right)\right]}{q}
$$

The following formula is used to obtain the ratio in analysis process of night environment factors of different categories and athlete psychological intervention effect:

$$
\omega_{k l}=\frac{f\left(e_{k}, z_{l}\right)}{\sum_{l=1}^{N} f\left(e_{k}, z_{k}\right)}
$$


The following formula is used to establish relevance model of night environment factors of different categories and athlete psychological intervention effect:

$$
e_{k}=\frac{\sum_{l=1}^{q} \omega_{k} z_{l m}}{\sum_{l=1}^{q} \omega_{k}}
$$

\section{Experiment results and analysis}

In order to verify the effectiveness of improved algorithms, there is the need for an experiment. Simulation software matlab is used to construct the experiment environment. In order to keep generality, in the process of the experiment, the traditional algorithm and improved algorithm are adopted for estimated results. The influence of two kinds of nocturnal environment for athletes psychological intervention is not same, but have same representative.

Figure 1 can describe influence variable statistical property caused by instantaneity and disorder of psychological changes in night environment of different algorithms:

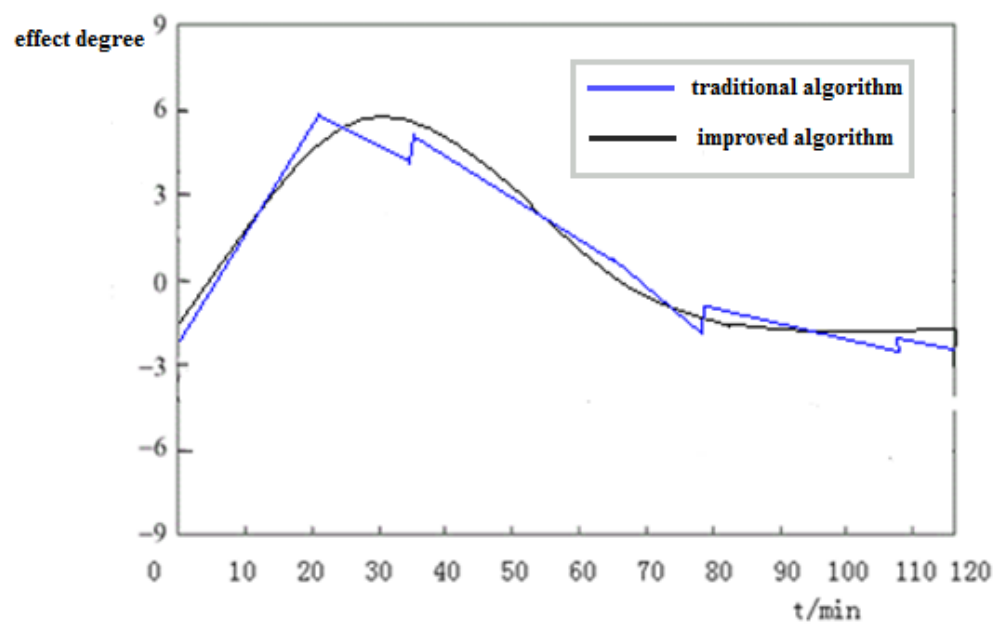

Fig. 1 Effect caused by night environment psychological changes in the athletes' psychological intervention effect degree

It can be learnt from Figure 1, the dynamic time series trend of night environmental psychology of two algorithms is similar, the values of all variables are on the rise, then decline. However, it can be seen from the above figure, different algorithms have a large difference for athletes' psychological intervention effect wave path. The smoothness of the improved algorithm is best, and the smoothness of the traditional algorithm is relatively low.

In order to verify the difference of different algorithms for athletes psychological intervention influence analysis caused by nocturnal environment, in the table below summarizes the statistic of night environment on mental. It can be learnt from the data in Table 1, the statistics of different algorithms have obvious differences, and the corresponding positive and negative symbols are not same.

Table 1 modeling results of different algorithms

\begin{tabular}{ccc}
\hline reference point & Traditional algorithm & Improved algorithm \\
\hline mean/\% & -0.06 & 0.67 \\
median/\% & 0.19 & 0.51 \\
maximum/\% & 5.32 & 6.62 \\
minimum/\% & -2.17 & -6.77 \\
standard deviation & 0.04 & 0.01 \\
deviation & 0.18 & -0.02 \\
peak & 7.5 & 5.6 \\
\hline
\end{tabular}


According to the experimental data, it can be learned that the proposed algorithm used for modeling the effect of night environment on psychological intervention for athletes, can affect the real effect of nocturnal environment for athletes psychological intervention, so as to process timely intervention, and improve the performance of athletes in the night environment.

\section{Conclusion}

In the process of analyzing the effects of night environment on athlete's psychological intervention, aiming at the problem of that the traditional algorithm not fully considers the instantaneous and disorder of night environmental psychological change, and resulting in the reduced accuracy of analyzing the effect of psychological intervention, a modeling method for the influence of night environment on athlete's psychological intervention based on the constrained fuzzy clustering algorithm is proposed. Firstly, the objective function of night environmental factors fuzzy clustering in the course of athletes in the sports is obtained to minimized transform. The weight and corresponding confidence coefficient of different nighttime environment factors are calculated, to establish the relationship model between night environmental factors and psychological intervention effect. The experimental results show that using the improved algorithm analyze the influence of night environment on athlete's psychological intervention, can improve the achievements of athletes at night.

\section{References}

[1] Wang Jianzhou, Zhou Chenglin, Sheng Zetian. The effect of psychological intervention on mental state of elite Greco Roman wrestlers before competition [J]. Journal of Tianjin University of Sport, 2008, 23 (3): 217-221.

[2] Tian Feng, Ou Wei, Zhang Hai, et al. The effect of biofeedback intervention on pre-competition psychological stress of college athletes [J]. Chinese Journal of behavioral medicine and brain science journal, 2014, 23 (2): 165-166.

[3] Zhang Shuting, Han Jie, Liu Wenkui. The psychological effects factors of tennis player in competition situation and intervention study [J]. Sports, 2010:34-35.

[4] Zhang Jianjie, Xu Nannan, Slig et al. Effect of Psychological Intervention on the Operation Performance of National Female Freestyle Skiing Athletes [J]. Journal of Tianjin University of Sport, 2014, 29 (2): 128-131.

[5] Lv You. Factors, Effects and Intervention Study of Short Track Speed Skaters Psychological Exhaustion [J]. Ice and snow sports, 2013, (4): 60-64.

[6] Zhang Zhongqiu. Development and Application of Sport Psychology in Competitive Sport Field [J]. Journal of Tianjin University of Sport, 2012, 27 (3): 185-191.

[7] Yao Jiaxin, Huang Zhijian. Current situation and Prospect of development of sports psychology in China [J]. Sports scientific research, 2010, 31 (6): 6-10.

[8] Shen Hejun. An empirical study of the psychological intervention for elite athletes [J]. Journal of Nanjing Sport Institute: Social Science Edition, 2011, 25 (6): 120-124. 